\title{
Treatment with valproate downregulates the Agtr2 mRNA in rat lungs
}

Kadri Seppa ${ }^{1}$, Anton Terasmaa ${ }^{1}$, Toomas Jagomäe ${ }^{2}$, Jürgen Innos $^{2}$, Eero Vasar $^{2}$, Mario Plaas $^{1 *}$

1Institute of Biomedicine and Translational Medicine, Laboratory Animal Centre, University of Tartu, 14B Ravila Street, Tartu 50411, Estonia

2Institute of Biomedicine and Translational Medicine, Department of Physiology, University of Tartu, 19 Ravila Street, Tartu 50411, Estonia

*To whom correspondence should be addressed:

Mario Plaas, Institute of Biomedicine and Translational Medicine, Laboratory Animal Centre, University of Tartu, 14B Ravila Street, Tartu 50411, Estonia. Phone: +372 502 3056, E-mail: mario.plaas@ut.ee

Email addresses:

kadri.seppa@ut.ee; anton.terasmaa@ut.ee; toomas.jagomae@gmail.com; jyrgen.innos@ut.ee; eero.vasar@ut.ee; mario.plaas@ut.ee

\section{Abstract:}

GLP1 receptor agonist liraglutide has been shown to upregulate ACE2 expressions in several animal studies and thereby mediate strong positive stress response ${ }^{1}$. On the other hand, two in silico studies suggest that valproate downregulates ACE2 and AGTR2 gene expressions ${ }^{2,3}$. In this study, we have evaluated how these two widely used drugs, liraglutide and valproate, change the expression pattern of RAS system genes in the rat lungs. Our results indicate that eight-day treatment with valproate significantly downregulates the gene expression of Agtr2, Mas1 and Agrtlb in the rat lungs. These effects are reversed by co-administration of liraglutide.

\section{Keywords:}

Valproate, liraglutide, GLP1 receptor agonist, ACE, ACE2, AGTR2, MAS1, AGTR1, lungs, renin-angiotensin system 


\section{Introduction}

The renin-angiotensin system (RAS) plays an important role in the control of body functions, including sodium and water content and blood pressure. Two main peptide hormones of the RAS system are the vasoconstrictor angiotensin II (Ang II) and the vasodilator angiotensin 17 (Ang-(1-7)), which have several receptors: Ang II type 1 and type 2 receptors (AGTR1 and AGTR2, respectively) and Ang-(1-7) MAS1 proto-oncogene, G protein-coupled receptor (Mas1). Angiotensinogen is the precursor of RAS peptides and is produced by the liver. In the blood stream it is converted into angiotensin I by renin which is released from the juxtaglomerulal cells of kidney due to the drop of blood pressure. Primarily in the pulmonary circulation angiotensin I is further processed by angiotensin-converting enzyme (ACE) into Ang II which binds to AGTR1 and AGTR2 receptors. Elevated Ang II production and AGTR1 activation contributes to elevated blood pressure, aldosterone secretion, water retention, proliferation, and pro-inflammatory and pro-fibrotic responses. AGTR2 receptor activation has the opposite effect, resulting in vasodilation. Ang II is further processed by angiotensin-converting enzyme 2 (ACE2) to a heptapeptide Ang-(1-7) which is an endogenous ligand for vasodilating Mas1 and AGTR2 receptors ${ }^{4,5}$. Besides vasodilatation, the activation of those two receptors results in nitric oxide release, subsequent hyperpolarization via activation of potassium channels, anti-inflammatory and anti-fibrotic responses, inhibits proliferation and finally leads to apoptosis ${ }^{4}$. Deregulation of the RAS system is implicated in the pathology of many diseases, including pulmonary fibrosis ${ }^{4}$, cancer $^{6}$, diabetes ${ }^{7}$, aging and neurodegeneration ${ }^{8}$.

The aim of this study was to evaluate the possible effects of pharmacological treatment with valproate and liraglutide (or their combination) on the regulation of gene expression levels of the key elements of RAS system. Therefore, the expression levels of Ace, Ace2, Agtrla, Agtr 1b, Agtr2 and Mas 1 were evaluated in the lung tissue of rats treated for eight days with two drugs shown to modify RAS system function: valproate, liraglutide, or their combination.

\section{Results}

To investigate how liraglutide, valproate or their co-treatment can modulate the function of the RAS system, we performed gene expression analyses of the lower lung lobe, extracted from rats after 8 days of treatment. The data were analysed using non-parametric KruskalWallis test followed by Dunn's multiple comparison test.

Gene expression analyses revealed no statistically significant changes in Ace (Figure 1a) and Ace 2 (Figure 1b) expression levels between treatment groups ( $\mathrm{p}>0.05$ ), although all treatments tended to increase Ace mRNA expression compared to saline group and valproate treatment tended to elevate Ace 2 expression. It has previously been shown that Ace 2 and Ace are co-expressed in the lung and Ace mRNA expression levels are higher compared to Ace $2^{9}$. Similarly, our results indicate that Ace mRNA expression levels are much higher than Ace2 mRNA level (Figure 1a, b).

In rodents two Agtr1a receptor subtypes are present, the Agtr1a and Agtr1b encoded by Agtrla and Agtrlb, respectively, and they appear to have differential regulation of their 
expression and possibly different functional properties ${ }^{10,11}$. Therefore, we performed gene expression analyses of both subtypes of the Agtr1 receptors.

Liraglutide had an increasing effect on Agrtla (Figure 1c) mRNA levels compared to saline group, but this difference was not statistically significant ( $\mathrm{p}>0.05)$, however, Agtrla gene expression was significantly downregulated after valproate treatment compared with liraglutide treatment $(\mathrm{p}<0.05)$.

Agtr1b (Figure 1d) expression was decreased in valproate treatment group compared to saline treated animals $(\mathrm{p}<0.05)$. Liraglutide had an increasing effect on Agrtlb mRNA levels compared to saline group, but this difference was not statistically significant $(\mathrm{p}>0.05)$. Additionally, valproate treatment had a significant decreasing effect on Agrtlb expression compared to liraglutide treatment $(\mathrm{p}<0.001)$, although valproate and liraglutide co-treatment reversed this decline $(\mathrm{p}<0.01)$.

Agtr2 (Figure 1e) expression was decreased in valproate treatment group compared to saline treated animals $(\mathrm{p}<0.01)$ similarly to Agtrlb expression. Liraglutide had a slight increasing effect on Agtr 2 mRNA levels compared to saline group, even though the difference was not statistically significant $(\mathrm{p}>0.05)$. Most importantly, valproate treatment had a significant decreasing effect on Agtr2 expression compared to liraglutide treatment ( $p<0.001)$, although in co-treatment liraglutide reversed the suppressing effect of valproate $(p<0.05)$.

Mas1 (Figure 1f) expression was decreased in valproate treatment group compared to saline treated animals $(\mathrm{p}<0.05)$ in the same manner as in Agtrlb and Agtr2 expression. Liraglutide had an increasing effect on Mas 1 mRNA levels compared to saline group, but the difference was not statistically significant $(\mathrm{p}>0.05)$. Additionally, valproate treatment had a significant decreasing effect on Mas 1 expression compared to liraglutide treatment ( $p<0.001)$, although valproate and liraglutide co-treatment reversed this decline $(\mathrm{p}<0.05)$.

In conclusion, valproate treatment downregulated the expression levels of Agrt1b, Agtr2 and Mas 1 basically to zero compared with saline treated animals and interestingly there is a clear pharmacological antagonism of this action by liraglutide (Figure 1d - f).

\section{Discussion}

In the lungs it has been shown that ACE is much more expressed compared to ACE2 2 . Similarly, our results indicate that Ace mRNA expression levels are significantly higher than Ace 2 mRNA level (Figure 1e, b).

Liraglutide is a glucagon-like peptide-1 receptor agonist (GLP1 receptor agonist), a common medication used to treat diabetes mellitus type 2 and obesity ${ }^{12}$. GLP1 receptor agonists have been shown to regulate several cellular targets to reduce cellular stress. Importantly, GLP1 receptor agonist treatment was found to upregulate ACE2 expression ${ }^{1}$.

Valproate is primarily used to treat epilepsy and bipolar disorder and to prevent migraine headaches. The precise mechanism of action of valproate is unknown; however, its effects include alteration of GABA levels, blockage of voltage-gated sodium channels, and inhibition of histone deacetylases ${ }^{13}$. Recent results from two in silico studies suggest that valproate can 
downregulate Ace2 and Agtr2 gene expression ${ }^{2,3}$. Thus, valproate may modulate the function of RAS system.

Based on this knowledge, we investigated how liraglutide and valproate treatment affect gene expressions of key genes of the RAS system in rat lungs.

Eight-day treatment with liraglutide, valproate or their combination had no major effect on gene expression levels of Ace, Ace 2 or Agtrla mRNA in rat lungs (Figure 1a-c). However, valproate treatment resulted in robust downregulation of Agtr1b, Agtr2 and Mas 1 mRNA levels in rat lungs (Figure 1d-f): such effect of valproate was clearly pharmacologically prevented by liraglutide (see combination treatment group, Figure 1d-f). Furthermore, liraglutide treatment tended to increase the expression of these genes, however, these effects were statistically non-significant. These results are in agreement with the findings of in silico studies where the effect of valproate on modulation of Agtr 2 was predicted ${ }^{2}$.

In summary, our results indicate that valproate treatment down-regulates the gene expression of Agrt2, Agtrlb and Mas 1 in the rat lungs, and therefore valproate can play an important role in the modulation of the RAS system. 


\section{Materials and Methods}

\section{Animals}

For this study, 3.5-4-month-old outbred $C D \circledR$ (Sprague Dawley) rats were used (in house breeding, stock line originated from Charles River Laboratories). Outbred rat line was chosen for better translational value. The animals were housed in groups of four animals per cage under a $12 \mathrm{~h}$ light/dark cycle (lights on at 7 a.m.). Rats had unlimited access to food and water. Sniff universal mouse and rat maintenance diet (Sniff \#V1534) and reverse osmosispurified water were used. Experiments were performed between 9 a.m. and 5 p.m.

All experimental protocols were approved by the Estonian Project Authorisation Committee for Animal Experiments (No 165, April 3, 2020), and all experiments were performed in accordance with the European Communities Directive of September 2010 (2010/63/EU).

\section{Repeated liraglutide and valproate treatment}

The rats were randomly allocated to the liraglutide (LIR, $n=8$ ), valproate (VPA, $n=8$ ), liraglutide + valproate (LIR + VPA, $n=8$ ), or saline control group (SAL, $n=8$ ). The liraglutide dose was $0.4 \mathrm{mg} / \mathrm{kg} /$ day (Novo Nordisk, Denmark) and the valproate dose was $0.3 \mathrm{~g} / \mathrm{kg} /$ day (valproic acid sodium salt, Sigma-Aldrich P4543). Effective and safe doses of the drugs were chosen based on our previous studies ${ }^{22,23}$.

All drugs where dissolved in $0.9 \%$ saline and injected subcutaneously at the volume of 1 $\mathrm{ml} / \mathrm{kg}$. The control group received $0.9 \%$ saline (vehicle) subcutaneously every day at the volume of $1 \mathrm{ml} / \mathrm{kg}$. After eight days of treatment and within $4 \mathrm{~h}$ after the last injection the animals were killed with intraperitoneal injection of Euthasol vet (dose $300 \mathrm{mg} / \mathrm{kg}$ ). Lower lobes of the lungs were dissected, washed quickly with $0.9 \%$ saline and snap frozen in liquid nitrogen. Tissue samples were stored at $-80^{\circ} \mathrm{C}$ until analysis.

\section{RNA isolation, cDNA synthesis and gene expression analyses}

The lower lung lobe was homogenized using Precellys lysing Kit CK14 (Bertin Instruments) and Precellys homogenizer (Bertin Instruments). RNA from rat lungs was isolated from the lysate using Direct-zol RNA MiniPrep (Zymo Research), according to the manufacturer's protocol. First-strand cDNA was synthesized using random hexamers and SuperScript ${ }^{\mathrm{TM}}$ III Reverse Transcriptase (Invitrogen, USA). qPCR using TaqMan Gene Expression Assays, and Taqman Gene Expression Mastermix (Thermo Fisher Scientific) was used to analyze Ace (Rn00561094_m1), Ace2 (Rn01416293_m1), Agtrla (Rn02758772_s1), Mas1 (Rn00562673_s1), Agtr1b (Rn02132799_s1) and Agtr2 (Rn00560677_s1) expression. Relative quantification was performed using the $2-\Delta \mathrm{Ct}$ method, with Hprt1 (hypoxanthineguanine phosphoribosyltransferase; Rn01527840_m1) as an internal control.

\section{Data analysis}

The data were analyzed using GraphPad Prism version 5 software (GraphPad Software Inc., San Diego, CA, USA). $\mathrm{p}<0.05$ was considered statistically significant. The data are presented as the mean \pm SEM and were compared using nonparametric Kruskal-Wallis test followed by Dunn's multiple comparison test. 


\section{Acknowledgements}

This research was supported by grant PSG471 (Mario Plaas) from the Estonian Research Council.

\section{Competing interests}

The authors declare no competing interest.

\section{Authors' contributions}

M.P. conceived the study. M.P., E.V., K.S. and A.T. designed the experiments, M.P. directed the study. A.T. and T.J. performed animal experiments. K.S. performed gene expression analysis. K.S., M.P., A.T., and J.I. participated in analysis and interpretation of the data and writing of the manuscript. A.T. and M.P. wrote most of the manuscript. All the authors have read and approved the final version of the manuscript.

\section{Figures and Tables}
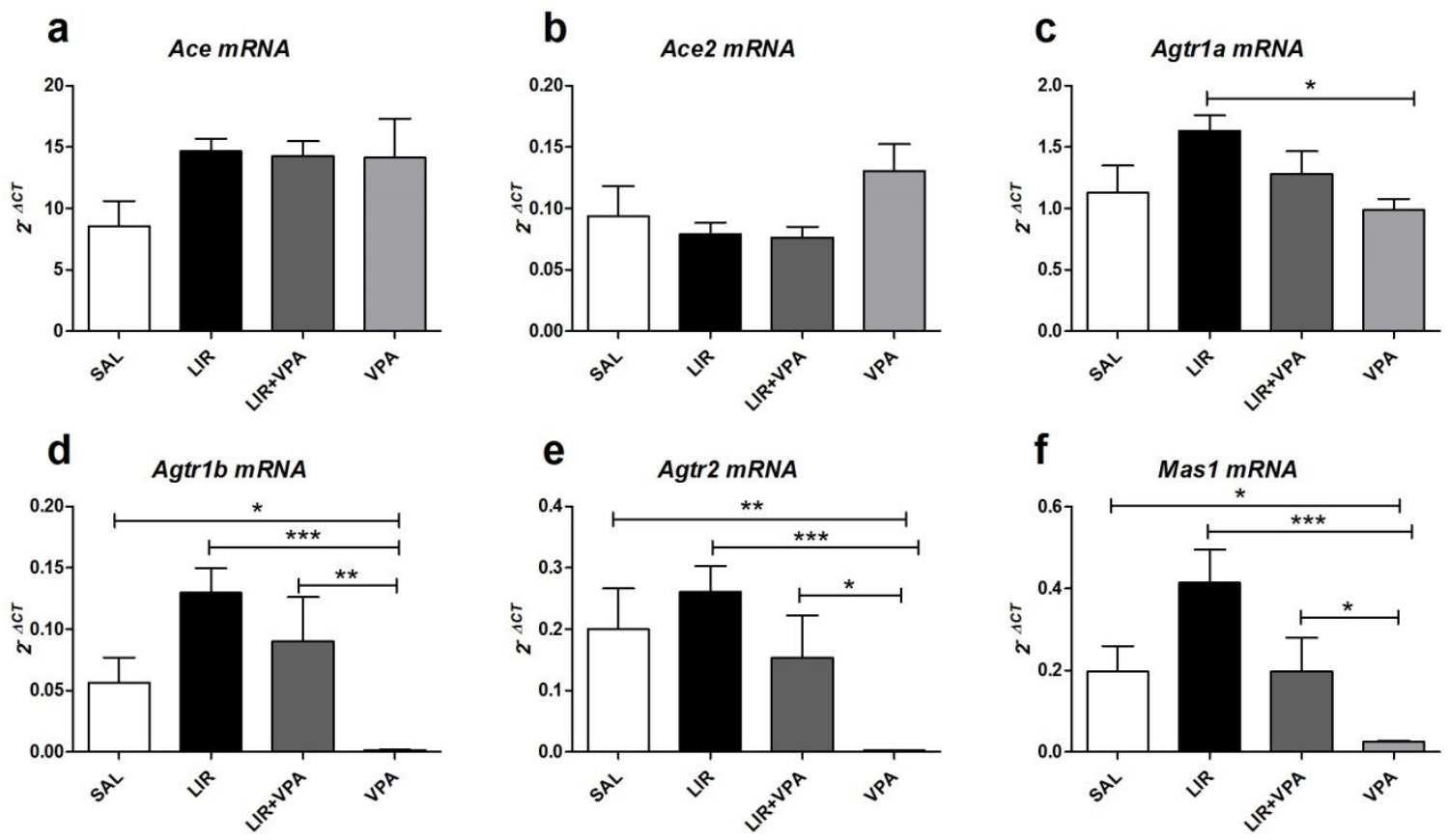

Figure 1. Lung gene expression analyses after 8 days of liraglutide (LIR), valproate (VPA) or liraglutide and valproate (LIR+VPA) co-treatment compared to saline group (SAL). Gene expression of (a) Ace; (b) Ace2; (c) Agtrla; (d) Agtrlb; (e) Agtr2 and (f) Mas1. The data were compared using nonparametric Kruskal-Wallis test followed by Dunn's multiple comparison test; $* p<0.05, * * p<0.01, * * * p<0.001$ compared to saline group. The data are presented as the mean \pm SEM, $n=8$ per group. 


\section{References}

1 Romani-Perez, M. et al. Activation of the GLP-1 Receptor by Liraglutide Increases ACE2 Expression, Reversing Right Ventricle Hypertrophy, and Improving the Production of SP-A and SP-B in the Lungs of Type 1 Diabetes Rats. Endocrinology 156, 3559-3569, doi:10.1210/en.2014-1685 (2015).

2 Cui, Q. et al. AGTR2, One Possible Novel Key Gene for the Entry of 2019-nCoV into Human Cells. Preprints, doi:10.20944/preprints202002.0194.v1 (2020).

3 Cui, Q. et al. Possible inhibitors of ACE2, the receptor of 2019-nCoV. Preprints 2020020047, doi:10.20944/preprints202002.0047.v1 (2020).

4 Ghadhanfar, E. et al. The role of ACE2, angiotensin-(1-7) and Mas1 receptor axis in glucocorticoid-induced intrauterine growth restriction. Reprod Biol Endocrinol 15, 97, doi:10.1186/s12958-017-0316-8 (2017).

5 Yue, X. et al. Nicotine Downregulates the Compensatory Angiotensin-Converting Enzyme 2/Angiotensin Type 2 Receptor of the Renin-Angiotensin System. Annals of the American Thoracic Society 15, S126-S127, doi:10.1513/AnnalsATS.201706-464MG (2018).

6 Perini, M. V., Dmello, R. S., Nero, T. L. \& Chand, A. L. Evaluating the benefits of reninangiotensin system inhibitors as cancer treatments. Pharmacol Ther, 107527, doi:10.1016/j.pharmthera.2020.107527 (2020).

7 Ribeiro-Oliveira, A., Jr. et al. The renin-angiotensin system and diabetes: an update. Vascular health and risk management 4, 787-803 (2008).

8 Labandeira-Garcia, J. L. et al. Brain Renin-Angiotensin System and Microglial Polarization: Implications for Aging and Neurodegeneration. Frontiers in aging neuroscience 9, 129, doi:10.3389/fnagi.2017.00129 (2017).

9 Riviere, G. et al. Angiotensin-converting enzyme 2 (ACE2) and ACE activities display tissue-specific sensitivity to undernutrition-programmed hypertension in the adult rat. Hypertension 46, 1169-1174, doi:10.1161/01.HYP.0000185148.27901.fe (2005).

10 Bogdarina, I. G., King, P. J. \& Clark, A. J. Characterization of the angiotensin (AT1b) receptor promoter and its regulation by glucocorticoids. Journal of molecular endocrinology 43, 73-80, doi:10.1677/JME-09-0036 (2009).

11 Kakar, S. S., Sellers, J. C., Devor, D. C., Musgrove, L. C. \& Neill, J. D. Angiotensin II type-1 receptor subtype cDNAs: differential tissue expression and hormonal regulation. Biochem Biophys Res Commun 183, 1090-1096, doi:10.1016/s0006291x(05)80302-x (1992).

12Patil, M., Deshmukh, N. J., Patel, M. \& Sangle, G. V. Glucagon-based therapy: Past, present and future. Peptides 127, 170296, doi:10.1016/j.peptides.2020.170296 (2020).

13 Tomson, T., Battino, D. \& Perucca, E. Valproic acid after five decades of use in epilepsy: time to reconsider the indications of a time-honoured drug. The Lancet. Neurology 15, 210-218, doi:10.1016/S1474-4422(15)00314-2 (2016). 

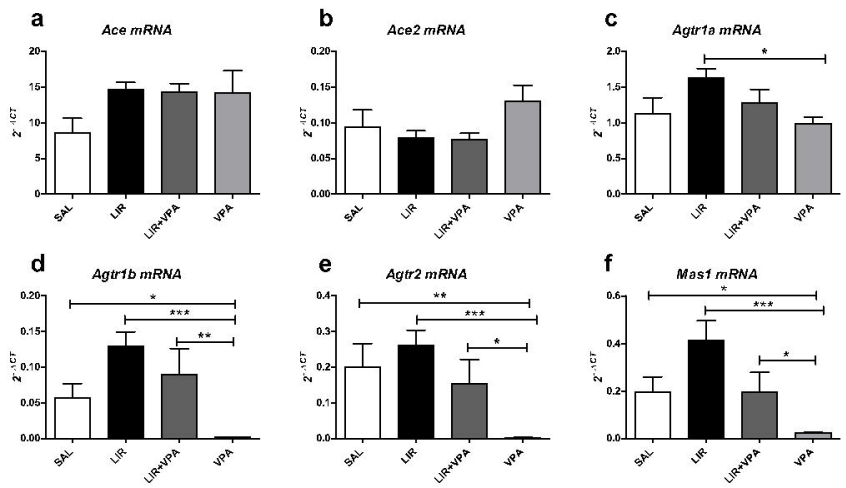